\title{
Heart Sound Segmentation Based on Personalized Gaussian Mixture Model and Convolutional Neural Network
}

\section{CURRENT STATUS: UNDER REVIEW}

BMC Medical Informatics and Decision Making $\triangle$ BMC series

Chun Dong Xu

Jiangxi University of Science and Technology

Jing Zhou

Jiangxi University of Technology

\1458311853@qq.comCorresponding Author

ORCiD: https://orcid.org/0000-0002-7945-3680

Dong Wen Ying

University of the Chinese Academy of Sciences

Lei Jing Hou

key Laboratory of Speech Acoustics and Content Understanding, Institute of Acoustics

Qing Hua Long

Jiangxi University of Sciences and Technology

DOI:

$10.21203 / \mathrm{rs} .2 .20414 / \mathrm{v} 1$

SUBJECT AREAS

Medical Informatics

KEYWORDS

Mel frequency cepstral coefficient, heart sound segmentation, heart sound classification, Gaussian mixture model, Convolutional neural network 
Abstract

Background: Heart sound segmentation is a long-standing problem in heart analysis, and it is mainly caused by noise interference and diversification of heart sounds. Faced with the challenging of heart sound segmentation, a more applicable segmentation model was studied. Methods: In this process, the optimal modified Log-spectral amplitude and wavelet were used to suppress the noise in the heart sound, and used the duration-dependent hidden Markov model based on personalized Gaussian mixture model (PGMM-DHMM) to segment the fundamental heart sound (FHS) and the nonfundamental heart sound (non-FHS). Then used the optimized Mel frequency cepstral coefficient (MFCC) to realize the classification of S1 and S2 heart sound frames through the Convolutional neural network (CNN) classifier, which can avoid the errors caused by the ambiguity of the time domain features. Results: PGMM-DHMM can segment FHS more effectively, and the accuracy is $94.3 \%$. The CNN classifier obtained the best results in the S1 and S2 classifications, the accuracy is $90.92 \%$, the precision of $\mathrm{S} 1$ is $90.76 \%$, the recall is $91.05 \%$, the $\mathrm{F}$-measure is $90.9 \%$, and the precision of S2 is $91.07 \%$, the recall is $90.79 \%$, the F-measure is $90.93 \%$. The final segmentation accuracy is $92.92 \%$. In addition, the experimental results further indicate that CNN has more robust performance when classifying abnormal S1 and abnormal S2. Conclusions: The PGMM-DHMM model can better segment FHS and Non-FHS. The optimization of MFCC improves the classification effect of S1 and S2, and the improvement effect by the CNN classifier is significant, especially for abnormal heart sounds. The proposed algorithm is better than other algorithms at this stage.

\section{Full Text}

Due to technical limitations, full-text HTML conversion of this manuscript could not be completed. However, the manuscript can be downloaded and accessed as a PDF.

\section{Figures}



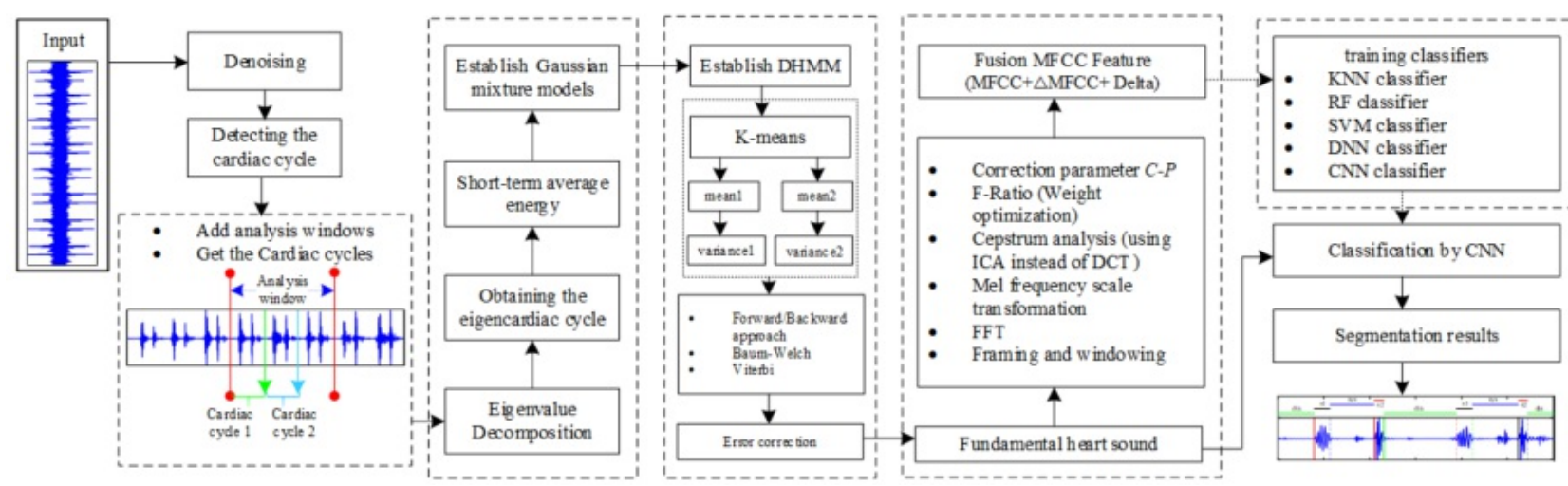

Figure 1

Detailed flow chart of the proposed algorithm
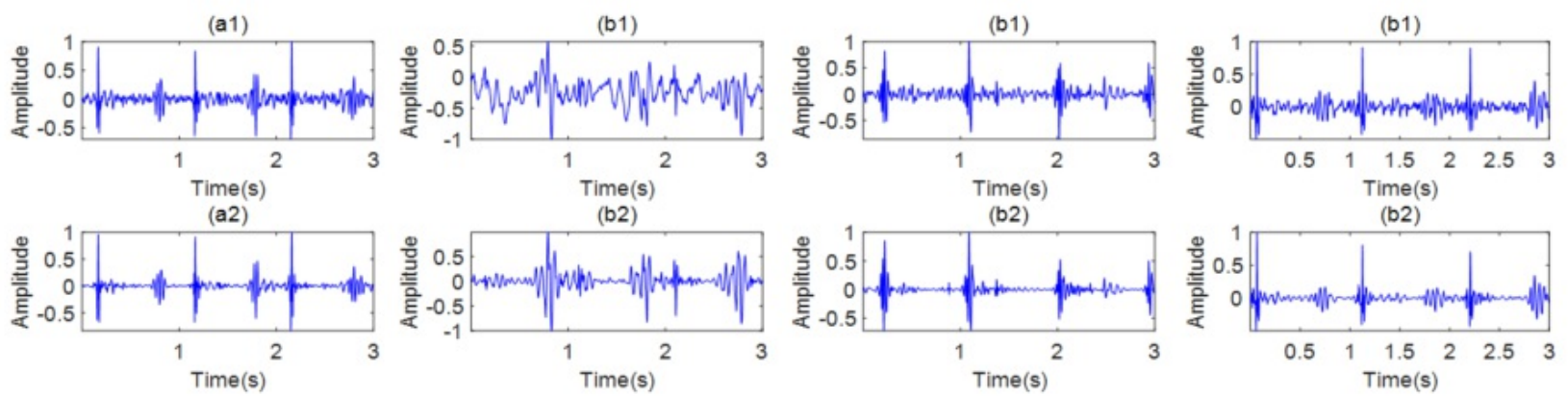

Figure 2

est results of noise reduction by OMLSA-W. (a1) The original a0001, (a2) The denoised a0001, (b1) The original b0087, (b2) The denoised b0087, (c1) The original c0018, (c2) The denoised c0018, (d1) The original d0052, (d2) The denoised d0052.

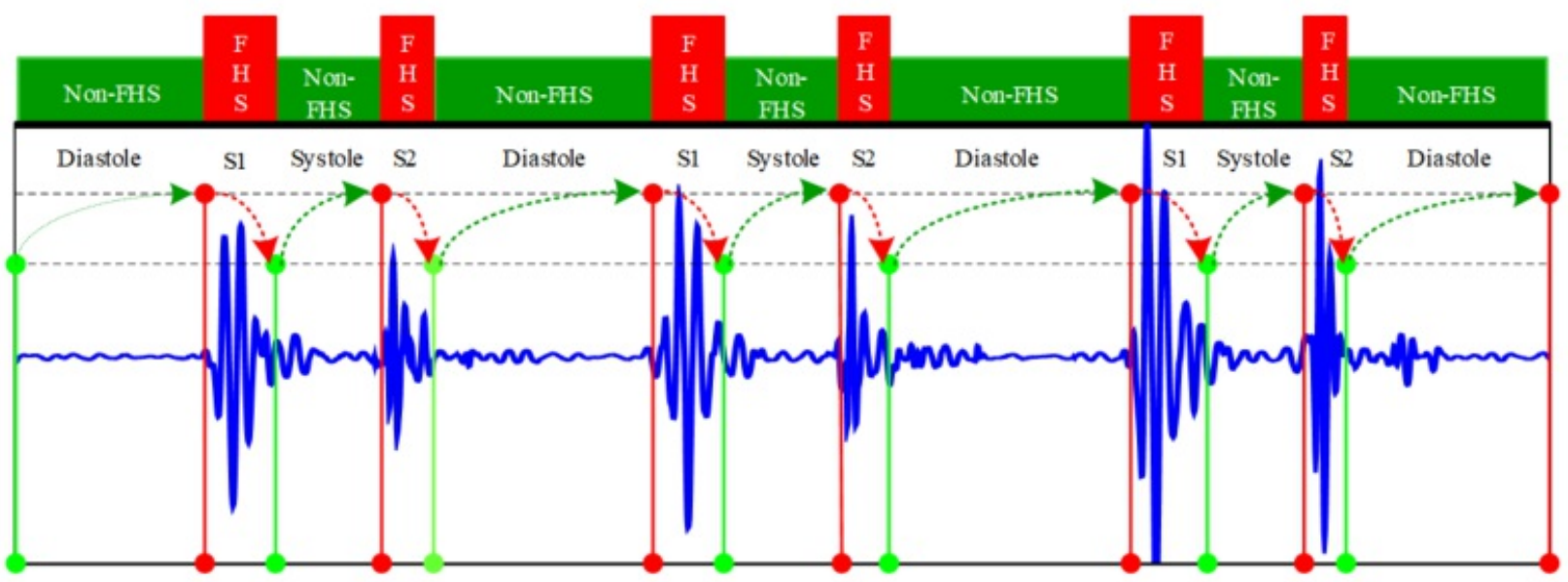

Figure 3

State transition between FHS and non-FHS 

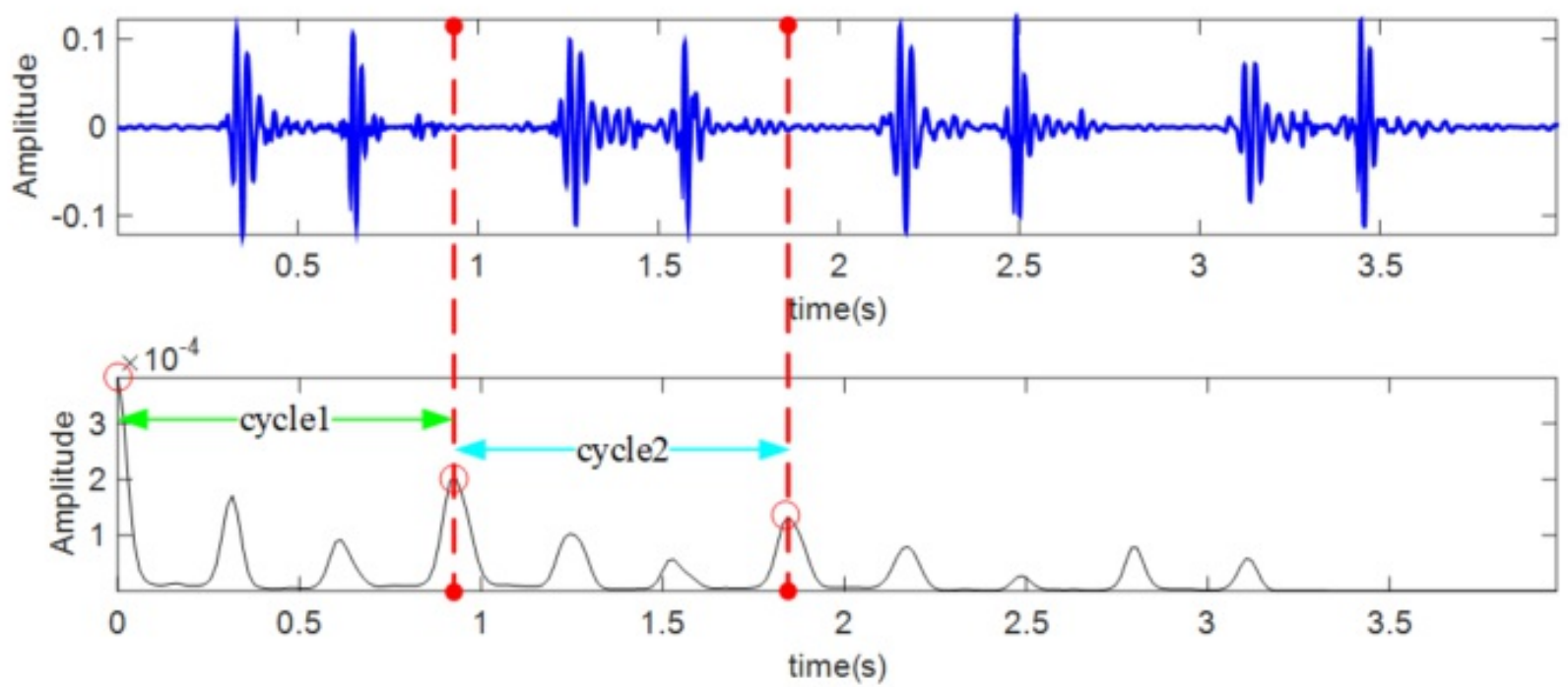

Figure 4

Detection of cardiac cycle

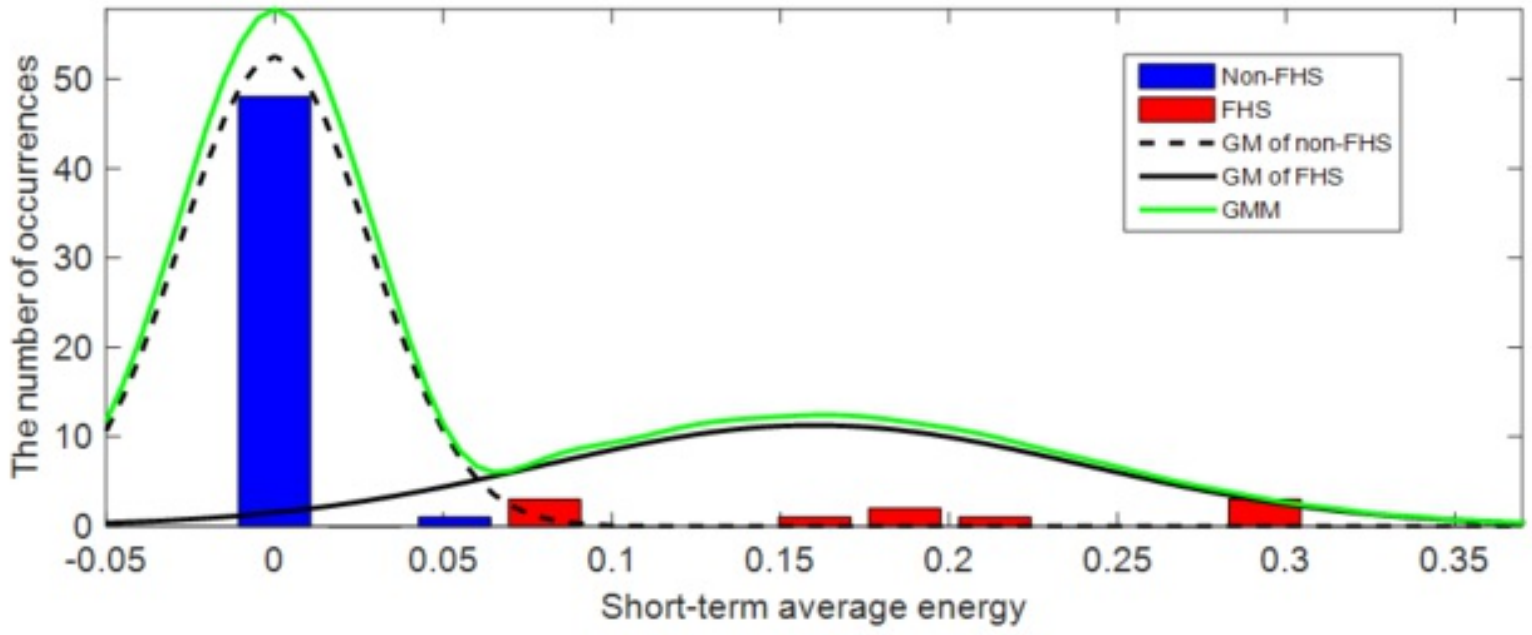

Figure 5

Gaussian modeling of eigenmode cardiac cycle 


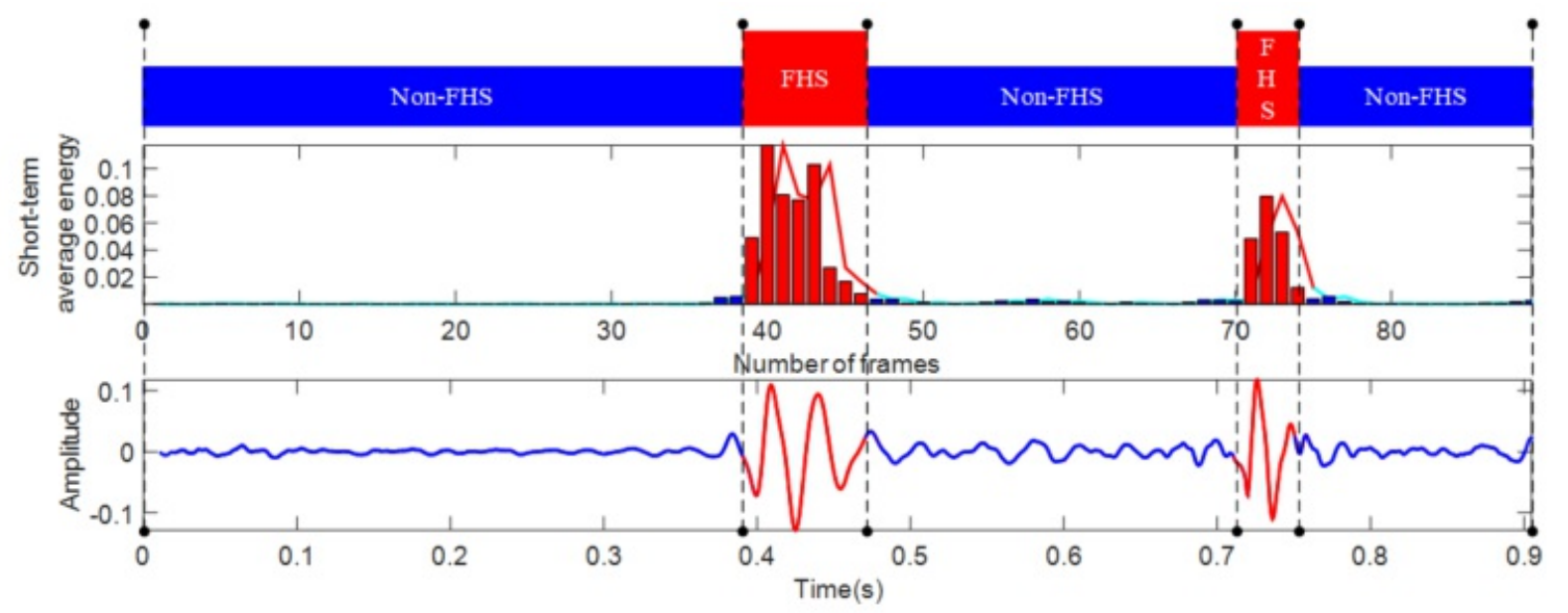

Figure 6

The cardiac cycle segmentation results of a0042

(a)

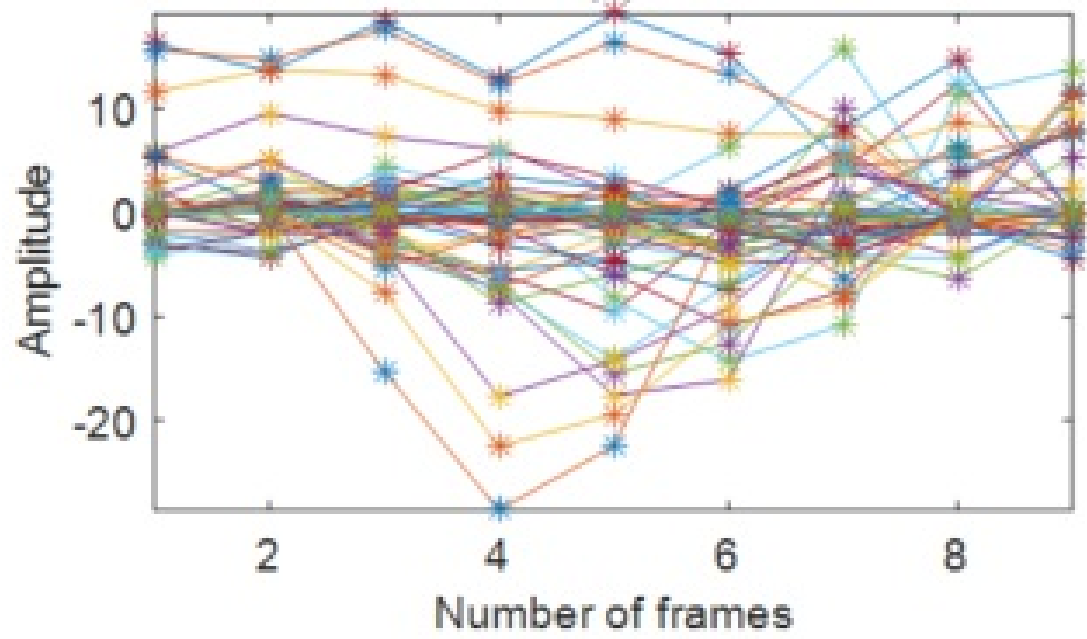

(b)

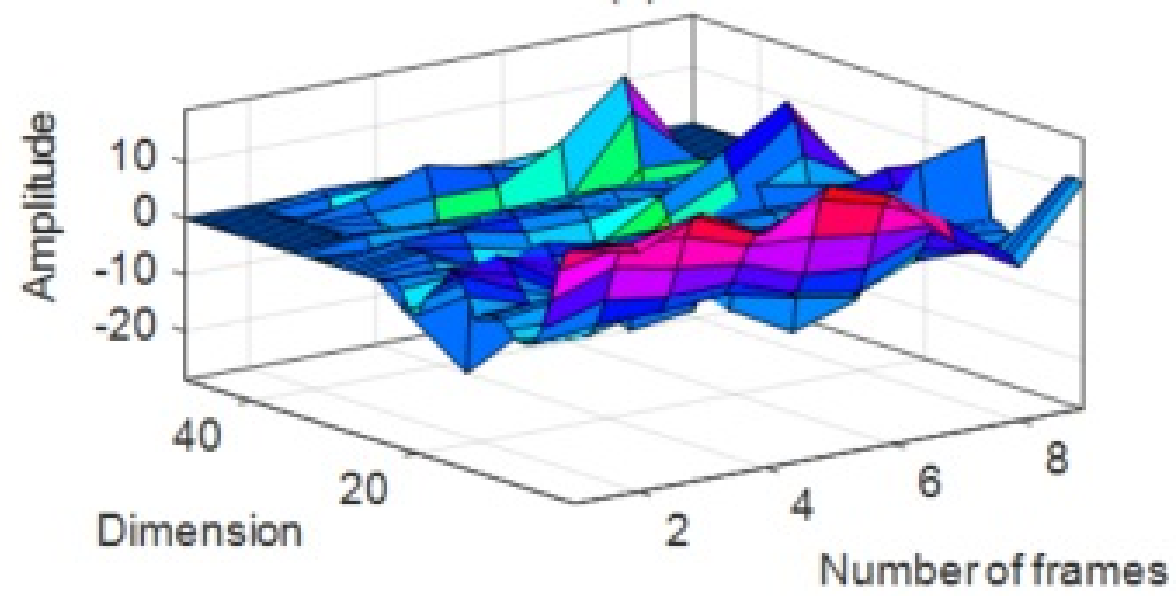

Figure 7

Visualization of MFCC: (a) Each dimension of MFCC, (b) 3D map of MFCC. 


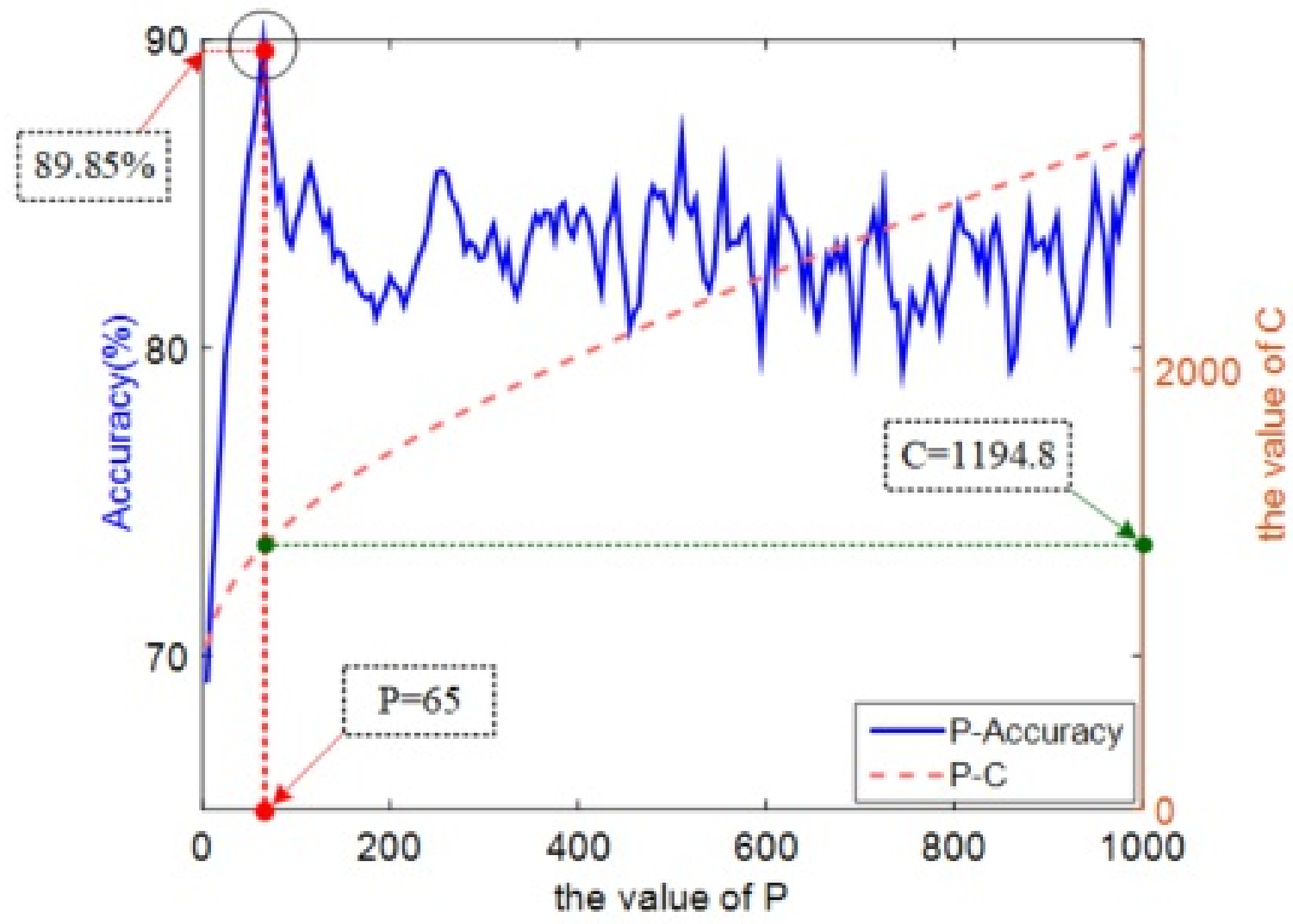

Figure 8

The test results of frequency scale conversion parameter correction

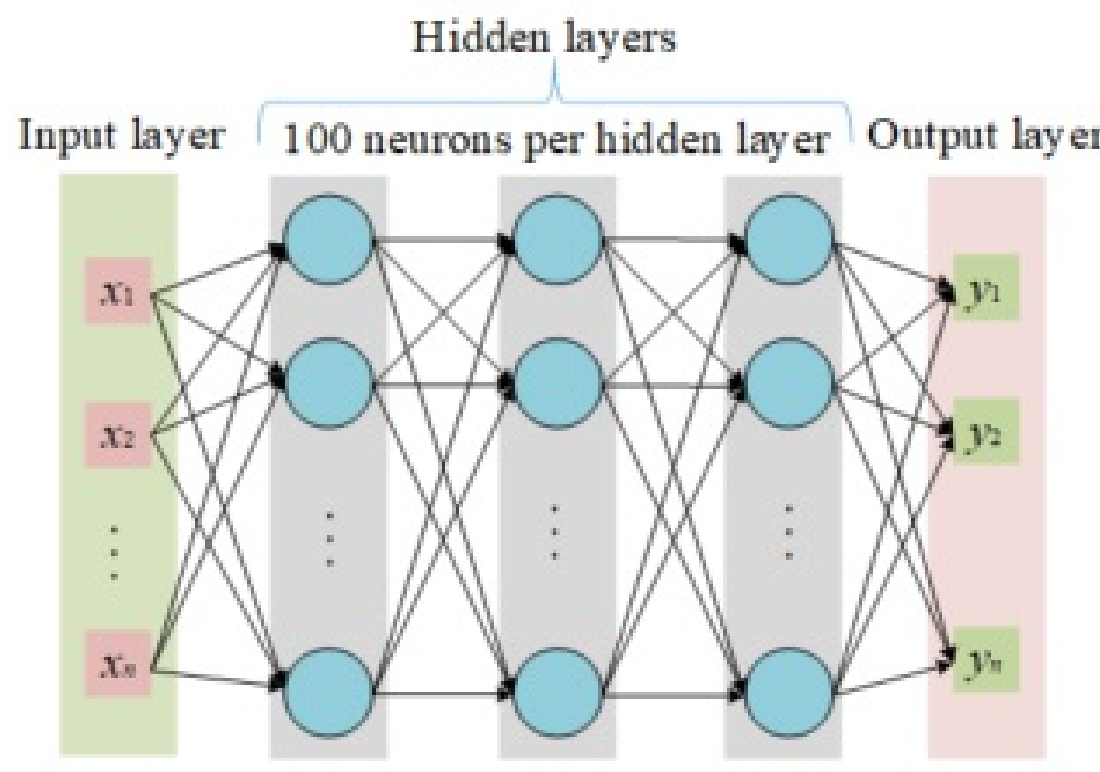

Figure 9

The structure of DNN classifier 


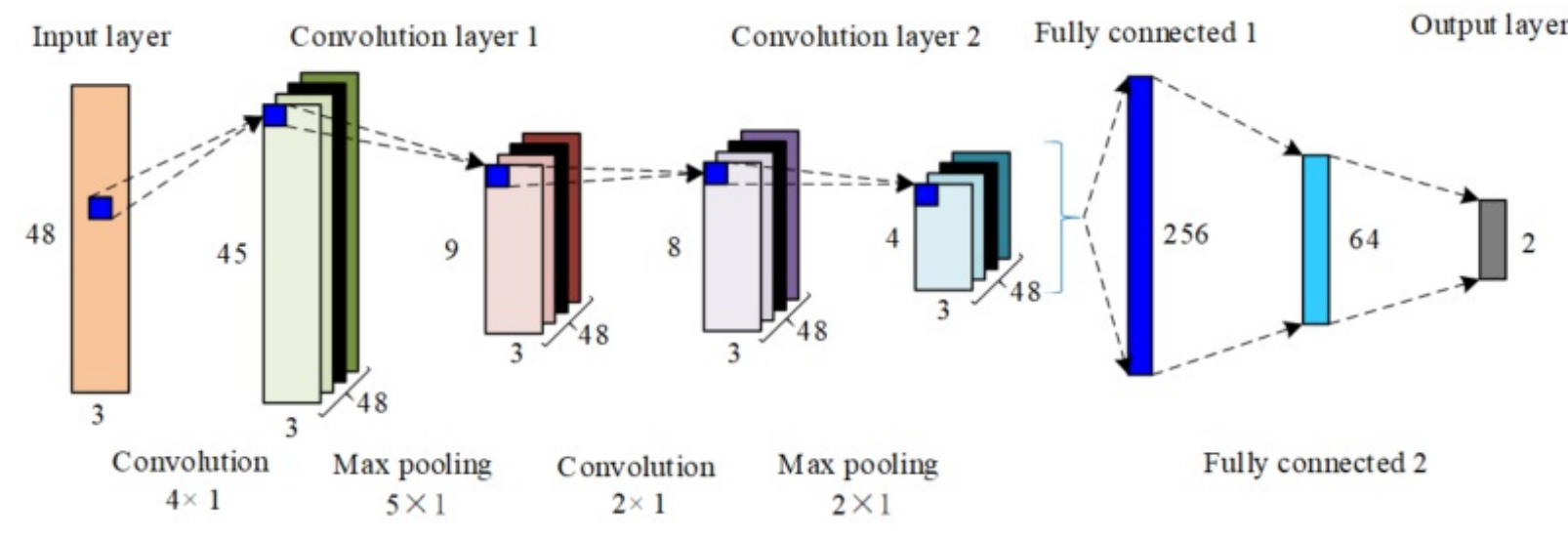

Figure 10

CNN classifier

(A)

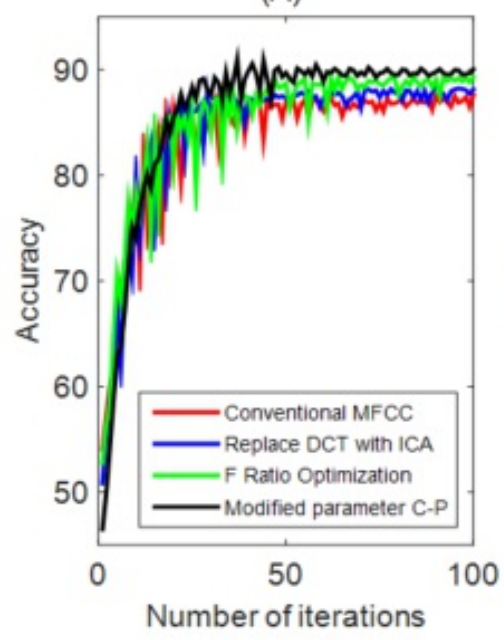

(B)

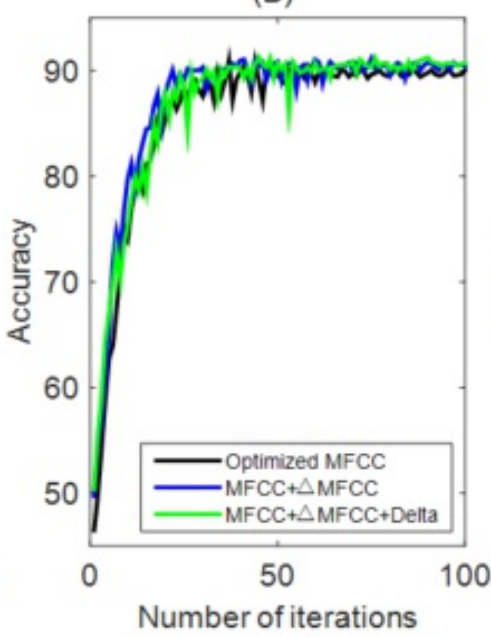

(C)

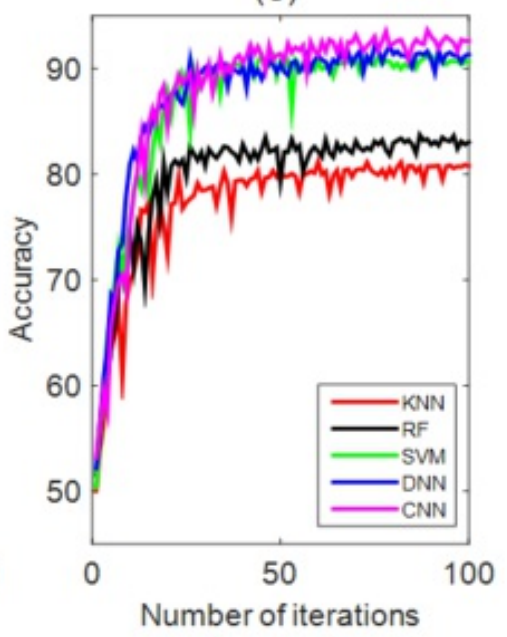

Figure 11

The training results, (a) Optimizing MFCC testing, (b) Fusion feature testing, (c) Classifier testing. 
(a)

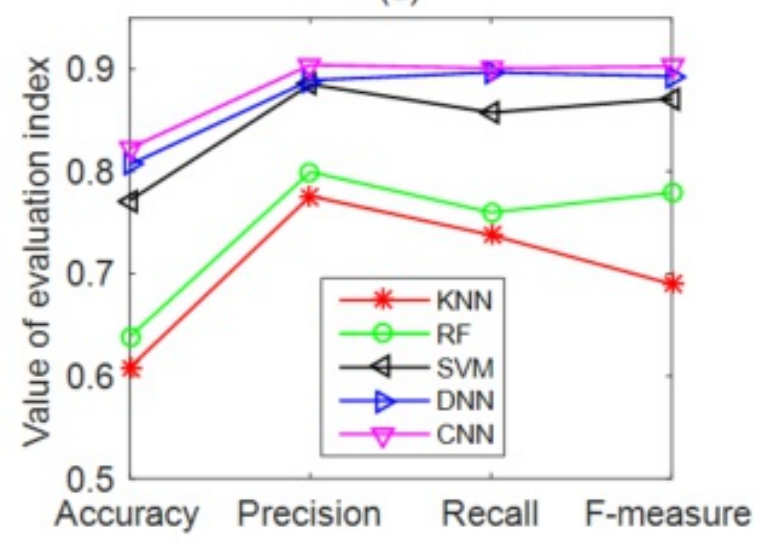

(c)

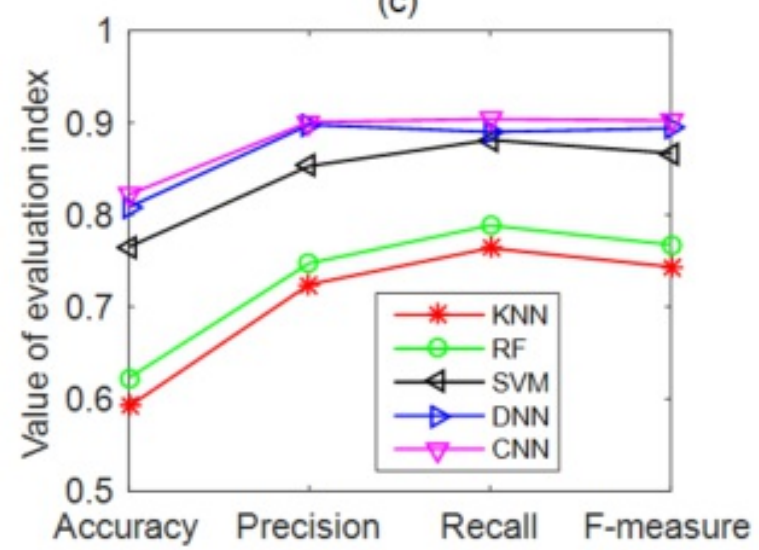

(b)

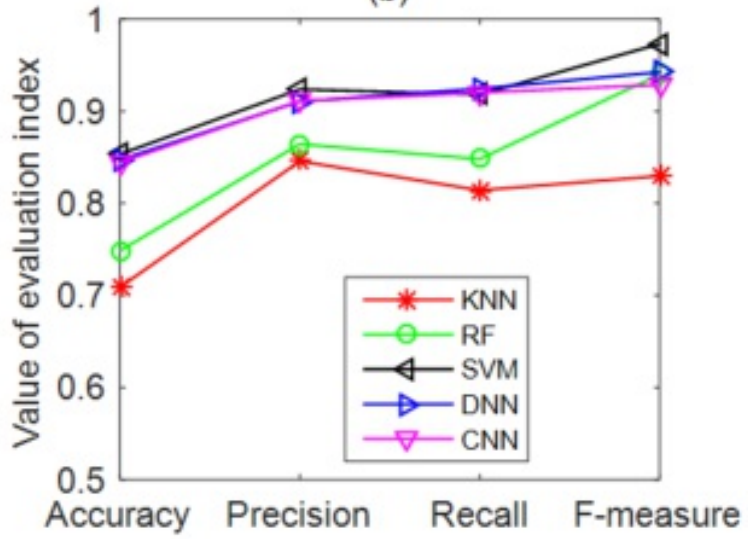

(d)

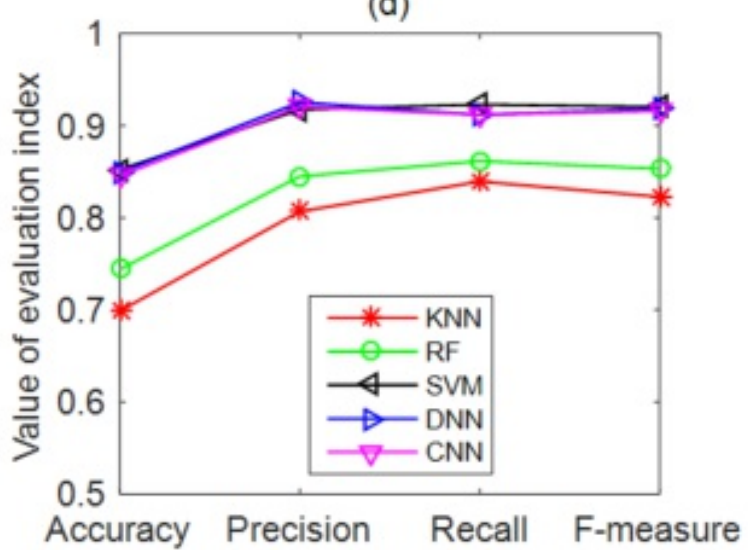

Figure 12

(a) The evaluation indexes of abnormality S1, (b) The evaluation indexes of the normal S1,

(c) The evaluation indexes of the abnormality S2, (d) the evaluation indexes of the normal 